\title{
La serie enumerativa en el cierre de los discursos
}

\author{
The listing series in the closing of discourses
}

\author{
Luís Cortés \\ Universidad de Almería, Departamento de Filología, Almería, España. \\ Correo electrónico: lcortes@ual.es
}

Los inicios y los cierres son las dos partes principales de un discurso. En nuestro estudio analizamos los cierres emitidos por los presidentes del gobierno español José Luis Rodríguez Zapatero y José María Aznar, en los “Debates acerca del Estado de la Nación” celebrados en el presente siglo. En todos ellos hemos podido vislumbrar unos mismos apartados y unos mismos mecanismos. De entre estos, sin duda, el que va a desempeñar un papel más relevante es el denominado por nosotros serie enumerativa, construcción que establece relaciones de linealidad, tónicamente simétricas, equifuncionales, desde el punto de vista sintácticopragmático, y con multifuncionalidad intencional (o interactiva). Dicha importancia va a venir condicionada por la capacidad de este mecanismo para expresar los rasgos propios de esta fase del discurso dominada por la exposición conclusiva, el énfasis argumentativo y el ornato.

Palabras clave: oralidad, discurso, cierre, discurso político, serie enumerativa.

The openings and closings are the two main parts of a discourse. In the present study we focus on the closings of the discourses produced by the presidents of the Spanish Government, José Luis Rodríguez Zapatero and José María Aznar, in the 'Debates on the State of the Nation' addressed in the present century. In all of them, the same sections and devices have been identified. Among these, particularly outstanding is what will be referred to here from a syntactic-semantic viewpoint as a "listing series", which is multipurpose in its functionality/ interaction potential. The priority of this element is in turn conditioned by the suitability of this device to convey the features of this stage of the discourse, in which a conclusive type of exposition, argumentative emphasis, and adornment are pre-eminent.

Key words: orality, discourse, closing, political, numbered series.

\section{INTRODUCCIÓN}

En Cortés (2010, 2011) aludíamos a la importancia que desde la tradición clásica se ha dado al cierre de un discurso. ${ }^{1}$ Es normal que la parte última de cualquier actuación

\footnotetext{
Ya señalaba Quintiliano (Fabi, 1991) que si bien el orador ha de tender en todas las partes del discurso a mantener vivos los sentimientos, esta preocupación deberá ser mayor al inicio de su intervención y, de manera muy especial, en la peroración final, parte dominada por la amplificación; cualquier orador no solo puede sino que debe poner el empeño suficiente para hacer su cierre más adornado y brillante que las demás partes.
} 
en público: concierto musical, representación teatral, mitin, etc. merezca un trato especial, pues influye sustancialmente en nuestra idea, positiva o negativamente, del evento. Inicio y cierre son los dos elementos fundamentales en todo discurso. Si con el primero se ha de buscar ganar el interés del público y empezar a crear una buena imagen, con el cierre se persigue el remate de esa buena imagen, lo que se consigue mezclando adecuadamente los ingredientes de su función: lo estético y lo eficaz. Esto quiere decir que un discurso en público deberá acabar con una conclusión o con un resumen claro y concreto de lo dicho previamente, pero en uno u otro caso irá acompañado de otra parte cuya función principal sea intentar mover el ánimo del auditorio. Y esto, repetido hasta la saciedad por los autores clásicos, lo aplicaban, por ejemplo, los oradores del siglo XIX, quienes cargaban de artificio -exclamaciones, paralelismos, pausas largas- sus cierres, aunque también de concreción y determinación. De Gracia (2001: 185) se refiere de esta manera al final del discurso que emite Castelar contra la esclavitud, en las cortes españolas, en junio de 1870:2

El final del discurso llega con extensos períodos limitados por exclamaciones. Castelar quiere lograr con ellos intensificar al máximo las emociones y los sentimientos de sus oyentes. Las curvas de entonación arrasan a la frase enunciativa y busca, desesperadamente, a través del halago sumar voluntades a su deseo.

En la búsqueda de ese ornato y solemnidad propios de los cierres de los discursos, los hablantes en general y los políticos en particular suelen usar determinados mecanismos oratorios; entre ellos: a) el alargamiento de las pausas; b) la entonación; c) la anáfora, que les permitía reforzar una idea o argumento mediante la repetición de una misma palabra o frase al inicio de diferentes actos discursivos; $d$ ) la concessio, figura que consiste en conceder parte de la razón al adversario en el asunto sobre el que se está discutiendo; en tanto que recurso retórico, este reconocimiento parcial de lo dicho por el otro, solo tiene por objeto intensificar la importancia del resto de aspectos en los que no se acepta la opinión del oponente, o e) el políptoton, con el que nuestros líderes juegan con una palabra empleando algunas de las varias formas que permite el cambio de los morfemas flexivos. Los políticos, puesto que son de los que vamos a tratar en este artículo, usan constantemente estrategias discursivas para que hagan más creíble lo que dicen y, por otro lado, puedan captar mejor al auditorio. Evidentemente que junto a los recursos referidos hay otros muchos, pero posiblemente ninguno más repetido e importante que la serie enumerativa. Y de ella vamos a hablar en el presente artículo.

Para su análisis, nos vamos a valer de algunos de los "Debates en torno al estado de la Nación” (en adelante DEN) que se celebran, anualmente, en España y que generan siempre un gran interés, pues en ellos gobierno y oposición abordan los asuntos políticos más importantes del país. ${ }^{3}$ Más concretamente, vamos a analizar los

Dicho final es éste: “¡Hijos de este siglo, este siglo os reclama que lo hagáis más grande que el siglo XV, el primero de la historia moderna con sus descubrimientos, y más grande que el siglo XVIII, el último de la Historia moderna con sus revoluciones! ¡Levantáos, legisladores españoles, y haced del Siglo XIX, vosotros que podéis poner su cúspide, el siglo de la redención definitiva y total de todos los esclavos!".

\footnotetext{
Instaurados por Felipe González en 1983 en la II Legislatura, su celebración es anual, y desde entonces solamente se han dejado de llevar a cabo en seis ocasiones, todas ellas por la convocatoria de elecciones generales; fue lo que ocurrió por última vez en 2008.
} 
discursos iniciales de lo presidentes del gobierno español en los debates celebrados en el presente siglo. ${ }^{4}$

\section{LAS SERIES ENUMERATIVAS Y SU PAPEL EN EL DISCURSO}

Aunque nuestra teoría de las series enumerativas, tipología, funciones, etc. está expuesta en Cortés, ed. (2008), sí creemos conveniente hacer un par de consideraciones.

La primera es que cuando hablamos de "serie enumerativa”, no hemos de asociar el adjetivo con el término enumeración tal y como ha sido empleado, para referirse a un tipo de marcador u ordenador del discurso, por algunos estudiosos como Auchlin (1981), Turco y Coltier (1988), Adam y Revaz (1989) o Adam (1990) aplicado al discurso francés, o Garcés (1996, 1997), al discurso español. Para estos autores, los marcadores u ordenadores enumerativos sirven para establecer las partes de un discurso e indican una sucesión: un antes -inicio- un después -desarrolloo un final -cierre-, o, simplemente, una ordenación espacio-locativa-temporal; de esta manera, facilitan la integración de los actos discursivos en sus correspondientes enunciados. Para nosotros, una serie enumerativa es un conjunto de elementos en relación, generalmente, de yuxtaposición, de adición o de disyunción, con los que se pretende mediante la reformulación parafrástica de un fragmento discursivo anterior, elemento común al que vamos a denominar matriz, la progresión temática del discurso materializada en distintos remas que se van asignando a un mismo tema provisional. El resultado será la presencia de otras tantas unidades discursivas (fragmentos, semiactos, actos, etc.) en relación textual de linealidad, tónicamente simétricas, equifuncionales, desde el punto de vista sintáctico-pragmático, y con multifuncionalidad intencional (o interactiva). No fue otra, por ejemplo, la pretensión del juglar del Poema de Mío Cid cuando relata la batalla para la toma de Alcocer y, en medio de esta descripción, queriendo insistir en lo grandioso del hecho, narra así al, probablemente, atónito auditorio:

(1) veriedes

tantas lanças premer e alçar, tanta adagara foradar e passar, tanta loriga falsar e desmanchar, tantos pendones blancos salir vermejos en sangre, tantos buenos vasallos sin sos dueños andar. (PMC 726-730)

o, por qué no, la de D. Quijote al valorar el estado de los caballeros andantes:

(2) no quiero yo decir, ni me pasa por pensamiento, que es tan buen estado el de caballero andante omo el del encerrado religioso; sólo quiero inferir, por lo que yo padezco, que, sin duda es más trabajoso y

El último debate hasta ahora celebrado fue en junio de 2011, en la sexta ocasión en que rendía cuentas el presidente Rodríguez Zapatero. En lo que va de siglo, los discursos iniciales de los presidentes del gobierno español en los DEN fueron emitidos por Aznar (2001, 2002 y 2003) y Zapatero (2005, 2006, 2007, 2009, 2010 y 2011). 


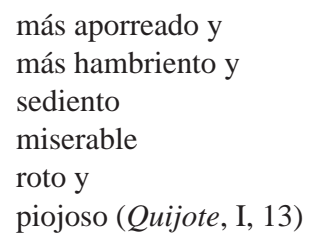

La segunda consideración tiene que ver con su eficacia discursiva. Sabemos que la adhesión de un interlocutor a una tesis presenta diferentes grados de intensidad; en el caso del discurso político, parece que se potencian todos los medios para enfatizar la convicción de las propuestas, de ahí la propensión de mecanismos que hagan más contundente la defensa de nuestra opinión. En el intento de convencer, la serie enumerativa es uno de los medios o marcas lingüísticas con mayor capacidad de convicción; el uso rítmico de cada uno de los elementos que componen las citadas series y, especialmente, la repetición de determinadas formas (mismas palabras, mismos tiempos verbales, idénticas estructuras, etc.) estimulan, más cuanto mayor sea el grado de ambos mecanismos, el efecto discursivo que persigue el comunicante: mover a los oyentes emocionalmente y contribuir a su convencimiento. Partimos de la idea, por tanto, de que determinados tipos de series, exceptuadas, por ejemplo, algunas listas de objetos, suelen cumplir la función interactiva de mover a la audiencia; sirven, por su estructura, para abarcar, de manera inmediata, varias razones (argumentos) en favor de una determinada conclusión, con lo que ello implica de énfasis discursivo. Por ello, si los discursos de los políticos, pasados y presentes, se valen de estas series, dada su innegable condición de mecanismo para elevar el peso argumentativo y la fuerza emotiva de cualquier orador, el hecho se dará en mayor medida en aquellos momentos, como son los cierres, en que también se persigue la solemnidad y el ornato. ${ }^{5}$ Podríamos decir que las series enumerativas son como marcadores de énfasis que sirven para indicar el alto grado de convicción con que el hablante quiere defender su punto de vista a la par que aporta, por sus repeticiones y estructuras, un grado mayor de formalidad y belleza al texto.

\section{LOS CIERRES DEL PRESIDENTE Y LAS SERIES ENUMERATIVAS}

\subsection{El cierre y sus partes}

Es curioso observar cómo todos los cierres presidenciales analizados, sean de una u otra ideología, responden a un mismo esquema. Comienzan con un vocativo, a continuación se habla de los ideales de partida; más tarde, de las realidades llevadas a cabo por el gobierno; se continúa con el tema de las cuestiones que quedan pendientes

\footnotetext{
Recordemos cómo ya Atkinson (1984) había señalado su efecto emotivo, hasta el punto de establecer una clara correspondencia entre su empleo y el aplauso espontáneo por parte de la audiencia que asiste a un mitin político. Más tarde, Geninasca (1987: 409), estudioso de la Semiótica, afirmó que: "Réduites au rang d'accessoires, dépourvues de vocation sémantique propre, les 'fleurs de rhétorique' auraient, au mieux, pour fonction de corroborer l'efficace persuasive des discours qui les cultivent”. Posteriormente, Tannen (1989) analizó el paralelismo como uno de los rasgos más importantes en la oratoria de Martin Luther King y Jessie Jackson. Es conocida la opinión de los tratadistas de la retórica, para quienes el paralelismo en general era un mecanismo a la hora de transmitir emoción; sabemos que se remonta a la retórica clásica la tradición de describir las estructuras textuales de los discursos políticos.
} 
para el futuro, y a continuación se suele dejar unos segundos para el halago, mediante la potenciación de los valores de España o de los españoles (libertad, dignidad, etc.); se termina con el agradecimiento por la atención. Si exceptuamos los ideales, de los que se prescinden en cuatro ocasiones -tres de ellas coincidiendo con los años de cierre del mandato- y el halago de los españoles, omitido en 2003, 2009 y 2011 y sustituido por otros temas, el resto de las cuestiones citadas se dan en todos los cierres, tal y como se puede ver en el cuadro siguiente:

\section{Cuadro $N^{o} 1$. Secuencias temáticas del cierre en los discursos presidenciales}

\begin{tabular}{|c|c|c|c|c|c|c|c|c|c|}
\hline Partes & 2001 & 2002 & 2003 & 2005 & 2006 & 2007 & 2009 & 2010 & 2011 \\
\hline Vocativos & S.P ${ }_{a}$ & S.P.P $/{ }^{1}{ }^{1}$ & S.P $/ \mathrm{a}$. & S. & S. & S.P/S. & S. & $\begin{array}{l}\mathrm{Sr} / \mathrm{Sra} \\
\text { Diputs }\end{array}$ & S. \\
\hline Ideales & $\mathrm{X}$ & $\mathrm{X}$ & & $\mathrm{X}$ & & & $\mathrm{X}$ & $\mathrm{X}$ & \\
\hline Realidades & $\mathrm{X}$ & $\mathrm{X}$ & $\mathrm{X}$ & $\mathrm{X}$ & $\mathrm{X}$ & $\mathrm{X}$ & $\mathrm{X}$ & $\mathrm{X}$ & $\mathrm{X}$ \\
\hline Futuro & $\mathrm{X}$ & $\mathrm{X}$ & $X$ & $X$ & $\mathrm{X}$ & $\mathrm{X}$ & $\mathrm{X}$ & $\mathrm{X}$ & $\mathrm{X}$ \\
\hline Halagos & $\mathrm{X}$ & $\mathrm{X}$ & $\mathrm{X}$ & $\mathrm{X}$ & & $\mathrm{X}$ & & $\mathrm{X}$ & \\
\hline Otros & & & & & $\begin{array}{l}\text { Terro- } \\
\text { rismo }\end{array}$ & & $\begin{array}{l}\text { Comp } \\
\text { social }\end{array}$ & & $\begin{array}{l}\text { Respe- } \\
\text { to }^{2}\end{array}$ \\
\hline Agradec. & $\begin{array}{l}\text { M.G., Sra. } \\
\text { Presidenta }\end{array}$ & $\begin{array}{l}\text { M.G Sra. } \\
\text { Presidenta }\end{array}$ & M.G. & M.G. & M.G. & M.G. & M.G. & M.G. & G. \\
\hline
\end{tabular}

1 S.P = Sr/a Presidente/a. S.= Señorías.

2 En 2011, conocida ya la renuncia del presidente Zapatero a presentarse en las próximas elecciones, dedicó el último minuto y medio a mostrar su respeto a todos los grupos y su gratitud a aquellos que han colaborado.

\subsection{Vocativos}

El inicio del cierre con un vocativo de los denominados honorativos (Bañón, $1993)^{6}$ se da en todos los casos analizados. Hace años que Haverkate (1978: 47) aludió al empleo de estos vocativos en determinados momentos dada su validez a la hora de reforzar ciertas funciones ilocutivas: "One could think here of the relatively high frequency with which vocativos occur in warning, promises, requests, etc.” Es evidente que en el caso que nos ocupa se quieren ofrecer ciertas advertencias. Pensamos, por tanto, que los vocativos iniciales de cierre cumplen en el discurso una doble función: por un lado, es una deferencia a las personas que ocupan en ese momento el hemiciclo (Señorías, Sra. Presidenta, etc.); por otro, se quiere manifestar ante ellos el interés de lo que van a oír, por lo que podemos decir que esa segunda función se aproxima a la de oye, escucha, atiende, o sea como marcadores interactivos centrados en el oyente y cuya función es captar el interés de este (Cortés y Camacho, 2005: 170). En este punto estamos de acuerdo con Edeso (2005: 123) cuando señala que el vocativo se emplea en el discurso, básicamente, para indicar cortesía ya positiva, ya negativa; para expresar autorreflexión por parte del hablante;

\footnotetext{
El tema del vocativo hasta su plena incorporación al análisis del discurso se ocupó tanto de temas que en trabajos como los que ahora nos ocupan pueden no ser de nuestro interés (su integración o no en la dinámica oracional) como de otros, más interesantes para el análisis de discurso (cuestiones relacionadas con su posición o función).
} 
y para tratar de implicar al auditorio en las palabras del emisor; bien es verdad que en este caso que nos ocupa, al ser un lenguaje protocolario, su uso atenúa en gran manera el valor cortés.

El presidente José María Aznar, en sus tres cierres (2001, 2002 y 2003), usa el vocativo Señora presidenta, seguido en dos casos de Señorías; hay, por tanto, en este empleo del doble vocativo un mayor deseo de implicación y un formulismo más cortés. Por el contrario, el presidente socialista, José Luis Rodríguez Zapatero, solo utiliza en una de sus seis actuaciones (2007) este doble vocativo: Sr. Presidente, Señorías; en el resto de las ocasiones en sus inicios de cierre implica únicamente a sus Señorías (2005, 2006, 2009 y 2011) y a las Señoras y Señores diputados (2010). ${ }^{7}$ Hay, en consecuencia, en estos pocos casos analizados, formas diferentes de vocativos en el líder de la derecha y en el de la izquierda, ${ }^{8}$ bien es verdad que en ambos casos, como corresponde al tipo de discurso que analizamos, se trata de vocativos empleados en situaciones formales, convencionales, y en cuyo uso sólo cabe pensar como manifestación, más que deferente, protocolaria; lo que ya Brown y Levinson (1978) denominaron cortesía formal o negativa. En todos los casos, no se podría negar un afán de atraer a los oyentes, sus señorías, a lo que se ha de decir por parte del hablante. De hecho, éste lo utiliza habitualmente precediendo al cambio de tema o a secuencias que considera importantes desde el punto de vista de su contenido.

Aunque no entendamos por qué, sabemos que en los discursos políticos analizados es infrecuente tanto el uso de ordenadores discursivos (en primer lugar, en segundo lugar...) para las distintas secuencias, como el empleo de comentadores, o sea de marcadores que señalen el punto concreto en que se encuentra el discurso (hasta aquí/ dicho esto; esto por lo que atañe a ... etc.). Una manera de rellenar esos huecos, en ocasiones entre secuencias y a veces enunciados, es mediante el uso del vocativo, que parece cumplir una nueva función: la de organizador. Siendo el cierre un cambio de secuencia importante, parece obvio pensar que este 'organizador' (vocativo) no pueda faltar.

Durante el cierre, usa otro vocativo: Señorías.

8 Los estudiosos del discurso han aceptado, junto al valor elocutivo, su carácter enfático o mitigador, si bien tales valores se suelen asociar a su posición media y, sobre todo, final, lo que apenas ocurre en el debate parlamentario, donde lo normal es que el vocativo, mayoritariamente Señorías, aparezca al inicio de un nuevo enunciado, lo que no implica una nueva secuencia. Sin considerar posición media los múltiples casos en que el vocativo va en medio de sujeto/verbo, verbo/complemento directo, etc., como ocurre en:

(i) Los resultados, señorías, están a la vista. Desde el 16 de abril de 2004 se han detenido 175 personas por su presunta vinculación con ETA, y por su relación con los atentados del 11-M se han producido 87 detenciones, 45 desde el 20 de abril de 2004, 23 permanecen en prisión como imputados (Zapatero, 2005).

(ii) En la buena situación de nuestro sistema de bienestar tiene mucho que ver también, señorías, el extraordinario aumento de la afiliación a la Seguridad Social; más de tres millones setecientos cincuenta mil nuevos afiliados desde 1996 (Aznar, 2002).

Muy extraño es en posición final:

(iii) Hemos optado por la segunda. Esa es la oposición útil, señorías. (Aplausos.) (Zapatero, 2001)

Algo más frecuente es si el vocativo final es Sr./a Presidente/a o el nombre del oponente:

(iv) Inseguridad, señorías, también en la calle, inseguridad ciudadana, inseguridad para los pequeños ahorradores que están viendo una evolución de la Bolsa muy preocupante. Hay muchas cosas que se le van de las manos, señor Aznar. (Zapatero, 2002). 


\subsection{Ideales}

El primer discurso analizado, Aznar (2001), comienza así:

(3) Señora presidenta, he anunciado a la Cámara los proyectos, iniciativas y reformas que el Gobierno se propone impulsar en los próximos meses. Vivimos en un momento de cambios relevantes en el panorama europeo. La entrada en circulación del euro afianzará la integración europea. La sociedad española será aún más abierta [la cursiva es nuestra].

Tras el obligado vocativo honorativo, se habla de una serie de propuestas, de proyectos, que nosotros hemos resumido con el epígrafe ideales, presentes en cinco de los nueve discursos analizados; es revelador que de los cuatro discursos en que faltan, tres sean finales de legislatura (2003, 2007 y 2011), donde ya no cabe hablar de ideales de partida, sino de realidades y de futuras propuestas.

El tratamiento de tales ideales es diferente en cada año; en el primer año de mandato, como hemos visto, son ideales que se han de cumplir; en el segundo, son ideales que se trazaron al llegar a la presidencia y en los que, obviamente, se ha avanzado:

(4) la recibí para cumplir un programa de reformas que nos acercara a un ideal ambicioso: alcanzar niveles de bienestar, desarrollo y proyección internacional similares a los de las naciones más avanzadas. Creo que el balance que presento hoy ante la Cámara refleja un esfuerzo intenso y un avance razonable en esta dirección (Aznar, 2002) [la cursiva es nuestra].

Igualmente pasa con Zapatero, si comparamos sus dos discursos iniciales (2005 y 2009):

(5) los gobiernos están para reconocer y aceptar que hay problemas, para tratar de entenderlos e interpretarlos y para intentar resolverlos, no para disimularlos o para ocultarlos, no para crearlos; en ningún caso para exacerbarlos. Los gobiernos están para dialogar, escuchar y respetar [la cursiva es nuestra].

(6) Señorías, hace cinco años aseguré que lo que define y caracteriza un proyecto socialista son las políticas sociales, las políticas destinadas a promover las condiciones de igualdad en el acceso a los bienes y servicios públicos, las políticas que garantizan la cohesión de nuestra sociedad y la hacen así más fuerte, más estable, más capaz y más justa [la cursiva es nuestra]. ${ }^{9}$

con el de 2010, en el que la necesidad, no lo prometido al inicio de la legislatura, lo enmascara de ideales que se han de llevar a cabo, e incluso que se han empezado ya:

(7) el esfuerzo colectivo que requiere la aplicación de las reformas económicas es necesario, tiene pleno sentido [...] Para preservar nuestro modelo de vida, nuestros valores y todas nuestras aspiraciones tiene pleno sentido este esfuerzo, y este esfuerzo hay que hacerlo ahora y de una vez para dejar atrás la crisis, lograr la recuperación, con fortaleza, que nos permita volver a la creación de empleo. Las circunstancias han cambiado (Zapatero, 2010).

\footnotetext{
En los ejemplos que sigan, la cursiva y negrita serán nuestras.
} 
No hemos de olvidar que en los debates, junto a los datos necesarios y decretos y leyes llevadas a cabo, así como a algunas realidades observables e irreductibles, en los discursos, Charaudeau (2009: 285), debido al contexto de persuasión, el político más que un razonamiento lógico con un objetivo explicativo o demostrativo, lo que hace es intentar mostrar la fuerza de la razón, por consiguiente, no se busca la verdad, sino la veracidad: se trata de decir no lo que es cierto, sino lo que se cree cierto y que los otros también deben creer cierto.

Por consiguiente, se impone al político una condición de simplificación argumentativa que lo lleva a proponer un razonamiento causal simple basado en creencias fuertes que se supone que la mayoría comparte (Charaudeau 2009: 285).

Estas creencias se deben fortalecer mediante unos mecanismos que lo hagan más emotivos e ineludibles. La serie es uno de ellos. Éste es uno de los motivos, el más importante por el que, sea uno u otro presidente, podemos ver cómo el estilo discursivo recurre a las series enumerativas, aunque es verdad que, al menos en los ejemplos que nos ocupan ahora, tales series son diferentes: las de Aznar (2001 y 2002) son series sencillas, ${ }^{10}$ tripartitas, cuyos elementos son nombres comunes sin complejidad alguna; además, sólo una serie en cada ideal. Son las denominadas series de lista, series que sirven para enumerar cosas, objetos, cualidades, etc., pero que al significar sus elementos, en estos casos, aspectos positivos, su uso se hace recomendable por aquello del valor de refuerzo entre los sumandos.

(8) he anunciado a la Cámara los

proyectos,

iniciativas $y$

reformas que el Gobierno se propone impulsar en los próximos meses (Aznar, 2001)

(9) nos acercara a un ideal ambicioso: alcanzar niveles de

bienestar,

desarrollo y

proyección internacional similares a los de las naciones...

(Aznar, 2002)

Quienes hacen los discursos socialistas emplean otros tipos de series, complejas ${ }^{11}$ y con mayor número de recursos retóricos: repeticiones, anáforas, etc. Veamos este primer discurso presidencial de Rodríguez Zapatero (2005) cuyo ideal de principios lo inicia con esta compleja serie enumerativa:

10 La serie enumerativa, entre otras diferentes tipologías establecidas en Cortés, ed., (2008) puede ser sencilla y compleja. Será sencilla, en casos como los citados ahora, en que la matriz o núcleo formal de la estructura genera dos o más segmentos que reformulan algún elemento temático contenido en la matriz.

11 Serán series complejas aquellas en las que de algún o algunos segmentos se derivan segmentos secundarios, terciarios, etc., que denominaremos abreviadamente como subsegmentos. 
(10) los gobiernos están (NIVEL 1)

para reconocer y (NIVEL 2)

aceptar que hay problemas,

para tratar de

entenderlos e (NIVEL 3)

para intentar

interpretarlos y

resolverlos,

(no)

para disimularlos $o$

para ocultarlos,

para crearlos;

para exacerbarlos.

(en ningún caso)

los gobiernos están [NIVEL 1]

para dialogar, [NIVEL 2]

escuchar y

respetar (Zapatero, 2005)

La anáfora, que se presenta en el discurso oral mediante la repetición de uno o más términos al inicio de una de sus unidades (enunciado, acto, microacto), tiene un fuerte contenido enfático; con su empleo se pretende reforzar mediante la repetición el contenido parcial de cada unidad precedida, así como el sentido global de todo el fragmento discursivo. La repetición anafórica de términos como los gobiernos están y para, se ve más reforzada aún con el uso, inmediatamente a continuación, exclusivo de los infinitivos, en series complejas. Vemos que del primer nivel de la serie, los gobiernos están, que al repetirse ya son elementos de la serie, se deriva un segundo nivel introducido con para + infinitivo repetido en múltiples ocasiones, y se cierra la complejidad con la derivación de un elemento terciario o sub-subsegmento: entenderlos e interpretarlos; resolverlos. En todos los casos se trata, mediante la expolitio, de ampliar aquella idea que el político pretende que permanezca por encima de las demás desarrollando su exposición.

Es más, para no salir de dicha expolitio y valerse del recurso de la repetición y de la serie enumerativa, se recurre a otra figura, la antítesis, al contraponer actos discursivos que expresan ideas de significación opuestas; por ejemplo: para intentar resolverlos, [NO] para disimularlos o para ocultarlos.

Los ideales de partida tras las necesarias medidas económicas que el presidente Zapatero se vio obligado a llevar a cabo se desarrollan en 2010 de una manera especial, tal y como hemos aludido más arriba. En efecto, es esencial justificar la necesidad de tal medida y recurre una vez más a la expolitio, para lo cual emplea el mecanismo más utilizado, en el discurso político, el empleo de complejas series enumerativas con repetición anafórica de determinados vocablos (es necesario para) a lo largo de ella:

(11) es necesario para (nivel 1)

que volvamos a crecer $y$ (nivel 2)

lo hagamos con

fuerza y (nivel 3)

sostenibilidad; 
es necesario para

que volvamos a crear empleo;

es necesario para

preservar el Estado de bienestar, asentado en esas cuatro

firmes columnas que son

la educación,

la sanidad pública,

las pensiones y ahora también

el sistema de dependencia;

es necesario para

mantener la política de incremento de becas que hemos hecho, [para] asegurar la renta de emancipación a los jóvenes que hemos puesto en marcha y hacer efectivo el derecho a una vivienda en condiciones asequibles;

es necesario para

defender la conciliación de la vida

familiar $y$

laboral, aplicando y extendiendo el permiso de [...];

es necesario para

proseguir la mejora de las pensiones mínimas como hemos hecho [...] y

es necesario, en fin, para

mantener la cohesión social en nuestro país, que es [...] (Aplausos)

[es necesario] para

preservar

nuestro modelo de vida,

nuestros valores $\mathbf{y}$

todas nuestras aspiraciones tiene pleno sentido este

esfuerzo

[Zapatero 2010]

El empleo de estas estructuras, con las que se inciden con énfasis en algunos elementos o ideas con un número menor de palabras, lo vamos a encontrar en todos los apartados del cierre.

\subsection{Realidades}

El apartado de realidades, de consecuciones llevadas a cabo, es el más variable en cuanto a su duración: son más reducidos los de Aznar que los de Rodríguez Zapatero. El primero sólo se refiere a cuestiones muy generales; así, en 2002 son éstas:

(12) estamos más cerca del pleno empleo;

invertimos más en aquello que es vital para nuestro futuro;

cuidamos mejor lo esencial de las personas y

fortalecemos las instituciones de la España constitucional (Aznar, 2002)

expuestas mediante una serie enumerativa sencilla de cuatro elementos que se inicia con un rasgo común: el tiempo y la persona verbal; en 2003, de forma parecida se vale de otra serie sencilla, tripartita con el artículo la+ sustantivo+adjetivo como sintagma reforzador de la serie: 
(13) la estabilidad política,

la prosperidad económica y

la proyección internacional

conseguidas en los últimos años ponen a nuestro país [...] (Aznar, 2003)

La exposición de las realidades por parte de Zapatero es mayor, especialmente en los tres últimos debates, cuando la situación económica empeoraba. El presidente aprovecha los cierres para enumerar de forma más explícita los logros de su gobierno. Así, en 2009 nos ofrece esta compleja serie enumerativa, cuyos elementos iniciales hace preceder del verbo ser (es, son), con función tanto enfática como cohesiva:

(14) Ese compromiso se ha ido haciendo realidad:

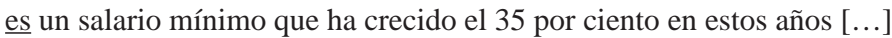
son las pensiones mínimas, que han crecido al 31 por ciento [...] (Aplausos); son las más de 650.000 personas en situación de dependencia que por primera vez han visto reconocida su situación y perciben prestaciones $y$

ayudas de la sociedad;

son los más de 1.500 .000 jóvenes que disfrutan hoy de una beca, 300.000 más [...]; son los 100.000 jóvenes que perciben la renta de emancipación para la ayuda [...]; son los 350.000 varones que han disfrutado del permiso de paternidad desde 2007 o las 800.000 familias que han percibido 2.500 euros por el nacimiento de [...]

son pues millones

de jóvenes,

de personas mayores,

de discapacitados, de dependientes,

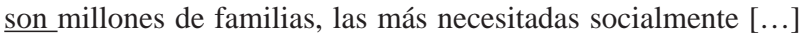
(Aplausos)

(Zapatero, 2009)

Igualmente ocurre en 2011, cierre con el que el presidente, a pesar de la situación económica, no da pie a que nadie dude de la capacidad de lo españoles y del país, pues:

(15) hemos superado en el pasado otros retos colectivos de una magnitud semejante y contamos con fortalezas indiscutibles

la renta per cápita de España

se sitúa en torno a los $\mathbf{2 5 . 0 0 0}$ euros y

se mantiene por encima del promedio europeo

la renta disponible es un 17 por ciento superior a la que había en 2003

$[\ldots]$

disponemos ya de

una de las mejores redes de infraestructuras del mundo. 
algunas de nuestras empresas lideran internacionalmente diversos sectores.

nuestra energía exportadora crece cada día.

hemos avanzado claramente

en la investigación y

en la innovación. [...]

comenzamos a reducir nuestros déficits educativos [...] y además

disponemos de un patrimonio y una riqueza sin comparación en nuestra

capacidad $[\ldots]$

disfrutamos de un nivel de servicios públicos muy estimable y

hemos logrado un considerable desarrollo de la política [...] [Zapatero, 2011]

En esta ocasión, el presidente opta, siempre en segunda persona del plural, por alternar dos tiempos verbales. Las series de Zapatero son más frecuentes y más complejas.

\subsection{Futuro}

En este apartado también las diferencias en los discursos de Aznar y Zapatero son considerables. Ahora es Aznar el que se muestra más explícito; como mecanismos, se vale bien de series enumerativas (2001, 2002), bien de amplios actos discursivos precedidos de marcadores textuales de progresión temática, tipo correlativo enumerador (en primer lugar, en segundo lugar, etc.), como ocurre en 2003.

En 2001 y 2002, decíamos, lo hace mediante una serie enumerativa sencilla, en cada caso, si bien la primera, con cinco elementos, recurre al artículo la + sustantivo como rasgo enfático, en tanto que en 2002, con siete elementos, la serie recurre al infinitivo como elemento unificador y de énfasis.

(16) tenemos que acometer con determinación los proyectos que ahora nos corresponde abordar,

como

la reforma de la justicia, las reformas educativas, la segunda reforma fiscal, la modernización de nuestras redes de comunicación e infraestruturas o la financiación autonómica (Aznar, 2001)

(17) un Gobierno que quiere llegar más lejos en los grandes retos de los españoles:

combatir el terrorismo, asegurar el ejercicio de las libertades, fortalecer la vertebración territorial, seguir creando empleo, modernizar nuestro país, incrementar el bienestar y

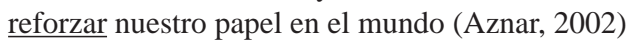


Diferente en cuanto a la forma y al fondo es la actuación de Aznar, en 2003. En ninguno de los discursos analizados se dedica tanto espacio, en el cierre, a hablar del futuro. Aznar emplea 487 palabras para plantear las tres tareas que, a su juicio, son prioritarias en el futuro, y que presenta - lo que no es habitual en este tipo de discurso- mediante ordenadores discursivos: "en primer lugar, afianzar la estabilidad de nuestro sistema institucional y fortalecer las funciones esenciales del Estado [...]; la segunda tarea que propone es afianzar las bases de nuestra prosperidad [...]; la tercera tarea es consolidar la posición internacional que el éxito de la España constitucional ha propiciado”. En la exposición de estas tres tareas, se muestra con frecuencia el mecanismo de las series enumerativas, especialmente en la segunda, casi toda formada por tal recurso. En la primera y segunda aparece un tipo de serie con estructura parecida, empleada por Aznar pero poco utilizada por otros políticos. Se trata de series que se apoyan sobre un elemento ya citado, el cual sirve de matriz, bien a través del pronombre correspondiente:

(18) En lugar de poner en cuestión los fundamentos del Estado, deberíamos ocuparnos de fortalecer sus

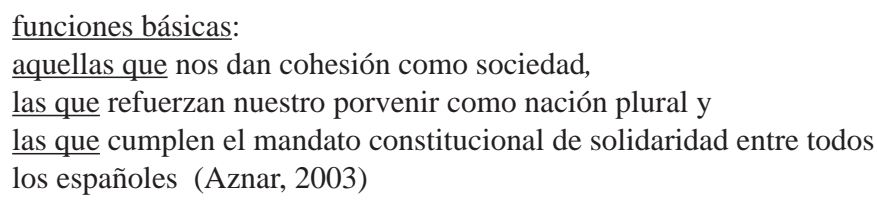

bien mediante el mismo vocablo:

(19) pero sólo podremos alcanzarlos si perseveramos en las reformas;

reformas para favorecer el empleo, para consolidar nuestro sistema de Seguridad Social o para aumentar la competencia; reformas a las que preceda el diálogo, buscando siempre [...]

(Aznar, 2003)

Es otra forma de reiteración de aquellos elementos que se quieren reforzar con objeto de que su incidencia entre los oyentes sea mayor; hemos de tener siempre presente que la serie enumerativa sirve para dar intensidad a cualquier argumentación. El tema del futuro en los cierres de Zapatero, especialmente en su primer mandato, decíamos, son inconcretos, genéricos y, por tanto, sin papel relevante alguno:

(20) junto a unas y otras, nuevas metas de progreso y de libertad aparecen en el horizonte de nuestra sociedad (Zapatero, 2005).

(21) reafirmo hoy mi determinación de trabajar para el fin de la violencia, para el fin de ETA [...]

Por mi parte comprometo nuevamente mi disposición al máximo diálogo para recorrer el camino a la paz.

(Aplausos) (Zapatero, 2006) 
(22) queda todavía un tramo por recorrer, hay todavía promesas por cumplir, objetivos que alcanzar y medidas que desarrollar [...]

Esa será la tarea del Gobierno en los próximos meses.

(Zapatero, 2007)

El fortalecimiento de las políticas sociales (2009), especialmente con las personas sin empleo, y la obligación de dirigir de la mejor manera, en el futuro, los cambios llevados a cabo por la necesidad (2010, 2011), son los temas a los que mira el presidente Zapatero en sus tres discursos de la IX legislatura:

(23) Quiero hoy reiterar, en nombre del Gobierno y del proyecto que represento, que este es nuestro principal compromiso y que, por tanto, mantendremos y fortaleceremos todas las políticas sociales que hemos ido conquistando en los últimos cinco años. Es mi mayor compromiso y también mi principal deber con los ciudadanos que nos dieron su apoyo electoral. Hoy extiendo ese compromiso de manera singular a los que en este momento más necesitan de la solidaridad y del apoyo de todos, las personas sin empleo. Ellas son destinatarias, con la misma fuerza y el mismo fundamento, de nuestro compromiso social acreditado, el que ha guiado toda nuestra acción política y la seguirá guiando en el futuro, para siempre (Zapatero, 2009).

(24) El momento es decisivo para engancharnos definitivamente al grupo de países más competitivos de Europa, al que siempre hemos querido pertenecer. Podemos negarnos a acometer esta modernización y correr el riesgo de languidecer, de quedarnos descolgados, o podemos afrontar la necesidad de los cambios para dirigirlos en la mejor dirección, hacer de la necesidad virtud y crear nuestro propio destino, porque este está en nuestras manos aunque nos cueste esfuerzos, y nos va a costar esfuerzos (Zapatero, 2010).

En este segundo discurso, se recurre a una serie enumerativa compleja, de tres niveles para reforzar la argumentación con que se quiere, una vez más, defender la necesidad de las reformas; solo su mantenimiento en el futuro podrá impedir el languidecer y quedarnos descolgados; en la serie, predomina el infinitivo, como elemento enfático y cohesivo:

podemos

negarnos a acometer esta modernización y

correr el riesgo

$$
\begin{aligned}
& \text { de languidecer, } \\
& \text { de quedarnos descolgados, o }
\end{aligned}
$$

podemos afrontar la necesidad de los cambios para

dirigirlos en la mejor dirección,

hacer de la necesidad virtud ${ }^{12} y$

crear nuestro propio destino

12 Zapatero pretende hacer ... de la necesidad ... virtud. 
A la obstinación del presidente en la defensa de la necesidad de sus impopulares medidas, así como de su persistencia en el futuro, gobierne quien gobierne, dedica el presidente buena parte del cierre en 2011, el último de su mandato:

(25) Pero necesitamos seguir haciendo un gran esfuerzo colectivo, con plena conciencia de que está en juego nuestro bienestar para las próximas décadas y que está en juego desmentir la afirmación de que vamos a tener una generación perdida. Mi opinión es que no vamos a perder una generación, la generación de los jóvenes que tienen una alta formación en nuestro país, pero ello va a exigir un gran esfuerzo colectivo, un gran sentido de la responsabilidad y una gran colaboración entre todos.

(Zapatero, 2011)

\subsection{Los halagos a España y a la sociedad española}

Salvo en tres ocasiones (2006, 2008 y 2010) en que Zapatero opta por dedicar los últimos instantes de su actuación a otros temas, en el resto de intervenciones el minuto final está dedicado a los valores bien de España como nación, bien de los españoles, lo que en la mayoría de los casos se expresa mediante series enumerativas con objeto de incidir a un mismo tiempo en varias cualidades. Así, tanto para Aznar como para Zapatero, hay que aprovechar el énfasis que da dicho mecanismo cuando se trata de los valores y cualidades que tienen los españoles, los votantes (libres, fuertes, sensatos, decididos, generosos, talentosos, capacitados, tesoneros, confiados en su futuro, tolerantes, modernos, etc.).

(26) Desde 1996 hemos querido impulsar

la libertad,

la confianza y

la solidaridad como los valores básicos de la sociedad española

(Aznar, 2001)

(27) Somos una sociedad

más libre,

más fuerte, más desarrollada y

más decidida a seguir avanzando, y esto creo que son los verdaderos rasgos distintivos del estado de nuestra nación (Aznar, 2002)

(28) He comprobado muchas veces en este año

cuánta sensatez,

cuánta generosidad,

cuánto talento albergan nuestras gentes (Zapateo, 2005)

(29) Si el país funciona, y funciona bien, es gracias a ellos, gracias a todos.

nuestros cimientos son sólidos y

nuestro horizonte esperanzador (Zapatero, 2005)

(30) Tengo una confianza plena en la sociedad española, en su capacidad, 
en su tesón, en sus valores, en su futuro (Zapatero, 2007)

(31) En este tiempo España se ha convertido en otro país, en un país del que podemos sentirnos orgullosos, en un país

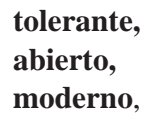

en el que se puede vivir a gusto.

(32) En este tiempo hemos sido capaces de vencer dificultades $y$ desafíos:

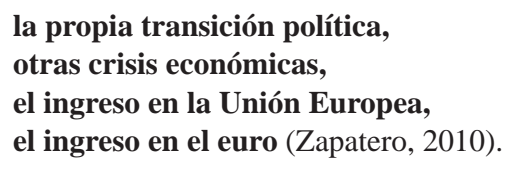

En todos los casos, son series sencillas de tres o cuatro elementos, salvo en 2010, en que Zapatero pretende hacer mayor el panegírico al querer referirse tanto a la cualidades presentes como a la capacidad de vencer las dificultades en el pasado. Por el contrario, en 2003 sólo una breve alusión a la confianza en el futuro de España y de los españoles.

\subsection{Otros temas}

En tres ocasiones (2006, 2009 y 2011) el presidente Zapatero no dedica su último minuto a la alabanza de los españoles sino a temas que considera de mayor importancia, y que desea que queden como preocupaciones esenciales de su gobierno en esos momentos: la solución del terrorismo (2006), el compromiso social de su gobierno en momentos tan críticos como los que vive el país, especialmente con los que han perdido el empleo (2009), y su manifestación de respeto hacia quienes han sido sus oponentes y sus aliados en estos años de mandato (2011); sabida es la renuncia del líder socialista a seguir como aspirante a la presidencia en las próximas elecciones. Es lógico que dedicara el último minuto de su discurso inaugural a tal motivo.

En estos fragmentos aún en su gravedad no faltan las series enumerativas con las que, por un lado, se enfatiza alguna idea, se hace más denso un argumento y, por otro, se agrandan el ornato y la solemnidad de cierre. Así, en 2006, Zapatero, que inicia ese final anunciando la necesidad de referirse al problema que más ha angustiado a la sociedad española desde la transición, el terrorismo, utiliza en dos ocasiones las series enumerativas: una cuando recuerda a las víctimas, recuerdo que no puede ser solo sentido, sino sentido y emocionado a la par que rotundo, para todo lo cual la serie enumerativa es un buen mecanismo de expresión: 
(33) Desde el recuerdo

sentido $y$

emocionado, $\mathrm{y}$

desde el apoyo a todas las víctimas del terrorismo [...] el derecho

al fin de la violencia,

a la paz $y$

a la esperanza de convivir juntos tan solo sometidos a los mandatos

\author{
de la libertad y \\ de la ley (Zapatero, 2006)
}

La otra, con una estructura paralelística, muy significativa y reforzadora, aparece cuando pide la comprensión de los españoles para acabar, mediante la paz con ETA, con esta desgracia que es el terrorismo:

(34) confío en que pueda ser una tarea de todos porque todos lo merecemos, confío en que todos actuemos con generosidad porque a todos nos [...] (Zapatero, 2006).

Pero, sin duda, donde la serie enumerativa alcanza su punto mayor de complejidad es en 2011, cuando el presidente se despide ${ }^{13}$ de sus señorías, lo que requiere, junto a la emotividad y contundencia expresivas, el ornato y la solemnidad. Para ello se vale de cuatro series enumerativas, en que no faltan repeticiones, políptotos, anáforas que consigue mediante la repetición del término respeto [de respeto (3), ese respeto (1) y mi respeto (1)] o anadiplosis, como ocurre en la serie tercera:

(35) [1] Hemos debatido sobre sobre la guerra $\mathrm{y}$

la paz,

sobre la discriminación y

la solidaridad,

sobre cómo terminar antes con la violencia de ETA

[2] Lo hemos hecho

en periodos de prosperidad y también

en esta época de

\author{
recesión y \\ grave preocupación social,
}

13 El fragmento es éste: "Hemos debatido sobre la guerra y la paz, sobre la discriminación y la solidaridad, sobre cómo terminar antes con la violencia de ETA. Lo hemos hecho en periodos de prosperidad y también en esta época de recesión y grave preocupación social, pero siempre hemos preservado la mayor riqueza que tenemos, la convivencia en paz y en libertad. De ahí que mi actitud siempre que he subido a hacer un debate sobre el estado de la Nación haya sido de respeto. De respeto, en primer lugar, a los ciudadanos, a quienes nos debemos; de respeto a esta institución, a la Cámara que encarna la soberanía popular; de respeto a todos los grupos y a sus señorías. Ese respeto es aún más profundo, para mí, a mi país, a España, sobre la que expreso mi más absoluta confianza en su futuro, y deseo expresar mi agradecimiento a todos los grupos políticos que, con crítica o reproches, vienen $-\mathrm{y}$ lo han hecho en unos momentos más y en otros menos- colaborando con el Gobierno. Mi respeto a todos los grupos y mi gratitud a aquellos que han colaborado. Y al Grupo Socialista, mi más profunda gratitud por su lealtad, por su compromiso y por su responsabilidad, el sentido de la responsabilidad que se espera de todos nosotros hoy, mañana. Gracias.” 
[3] De ahí que mi actitud siempre que he subido a hacer un debate sobre el estado de la Nación haya sido de respeto.

de respeto, en primer lugar, a los ciudadanos, a quienes nos debemos;

de respeto a esta institución, a la Cámara que encarna la soberanía popular;

de respeto

a todos los grupos $y$

a sus señorías.

[4] y al Grupo Socialista, mi más profunda gratitud

por su lealtad,

por su compromiso y

por su responsabilidad, el sentido de la responsabilidad que se espera de

todos nosotros

hoy,

mañana y

todos los días del futuro

\subsection{Agradecimiento}

Más ceremonioso se muestra el presidente Aznar, que el presidente Zapatero; el primero en 2001 y 2002 utiliza la forma muchas gracias, Sr. presidenta y muchas gracias, en 2003; en tanto, Zapatero utiliza esta forma última en todas las ocasiones, exceptuado en 2011, que se reduce a gracias, curiosamente el año en que su final de cierre es más emotivo.

\section{CONCLUSIONES}

El cierre es la parte más importante de un discurso. No obstante, las diferencias entre tales cierres, en los Debates acerca del Estado de la Nación, cuando son emitidos por el presidente o cuando quien los emite es el líder de la oposición, son grandes. En el primer caso, en los pronunciados por los presidentes, se puede ver una serie de apartados que hemos intentado vislumbrar en este artículo. Nada de ello ocurre en los formulados por los líderes de la oposición, como se analizará en otro trabajo.

Un tipo de mecanismo como la serie enumerativa, que establece relaciones de linealidad, tónicamente simétricas, equifuncionales, desde el punto de vista sintácticopragmático, y con multifuncionalidad intencional (o interactiva), va a desempeñar un importante papel en una parte del discurso, como es el cierre, en que se han de unir la concreción, el refuerzo del argumento, el ornato y la solemnidad. La función de tales series y su tipología guardan relación de dependencia con cada una de las partes en que hemos dividido los cierres de los presidentes analizados, con los temas tratados e incluso con los años en que transcurre el mandato de cada uno de los presidentes.

\section{OBRAS CITADAS}

Adam, J.M. 1990. Éléments de linguistique textuelle. Lieja: Mardaga.

Adam, J.M y F. Revaz 1989. "Aspects de la structuration du texte decriptif. les marqueurs d'énumération et de reformulation ”. Langue Française 81: 59-98. 
Atkinson, M., 1984. Our masters voices. The language and body language of politics. Londres: Routledge.

Auchlin, A. 1981. "Rèflexions sur les marqueurs de structuration de la conversation". Études de Linguistique Appliquée 44: 59-98.

Bañon, A.M. 1993. El vocativo, Propuestas para su análisis lingüístico. Barcelona: Octaedro.

Cortés, L. 2007, "Las series enumerativas en el debate político tras el atentado de Barajas. Zapatero y Rajoy frente a frente”. Oralia 10: 47-102.

Cortés, L. 2010. Comentario pragmático de comunicación oral. III. Una ponencia en un Foro. Madrid: Arco/Libros.

Cortés, L. 2011. "El plano secuencial y los debates en torno al estado de la nación. I. El discurso del presidente”. Círculo de Lingüstica Aplicada a la Comunicación 46: 3-50. Disponibe en http.//www.ucm.es/info/circulo/no46/cortes.pdf

Cortés, L. (ed.). 2008. La serie enumerativa en el discurso oral en español. Madrid: Arco/ Libros.

Cortés, L. y Mª.M. Camacho 2005. Unidades de segmentación y marcadores del discurso. Madrid: Arco/Libros.

De Gracia, A. 2001. "La persuasión en el discurso de Emilio Castelar”. J.A. Hernández Guerrero (ed.). Emilio Castelar y su época. Idelogía, retórica y poética. Cádiz: Universidad. 175188.

Edeso, V. 2005. "Usos discursivos del vocativo en español”. Español Actual 84: 123-142.

Fabi, M. 1991. Institutionis oratoriae libri Dvodecim. Oxford: University Press.

Garcés, M. ${ }^{\mathrm{P} P}$. 1996. "Los marcadores discursivos en español”. Gil y Ch Schmitt (coords.). Kohäsion, Koharenz, Modalität in Texten romanischer Sprachen. Bonn: Romanistischer Verlag. 125-147.

Garcés, M.P. 1997. "Procedimientos de ordenación en los textos escritos”. Romanistisches Jahrbuch 48: 296-315.

Geminasca, L. 1987. "L' enumeration, un problème de semiotique discursive”. Mélanges offerts à G. Hilty. Romania ingeniosa. Berna:Peter Lang. 407-419.

Haverkate, H. 1978. "The vocativo phrase in modern Spanish. A contribution to the study of illocutionary functions”. M. Zonneveld (ed.). Linguistics in the Netherlands. Lisse: Peter de Ridde. 46-62

Tannen, D. 1989. Talking voices. Repetition, dialogue and imagery in conversational discourse. Cambridge: Cambridge University Press.

Turgo, G. Y D. Coltier 1988. "Des agents doubles de l'organisation textuelle. Les marqueurs d’intégration linéaire ”. Pratiques 57: 57-79. 